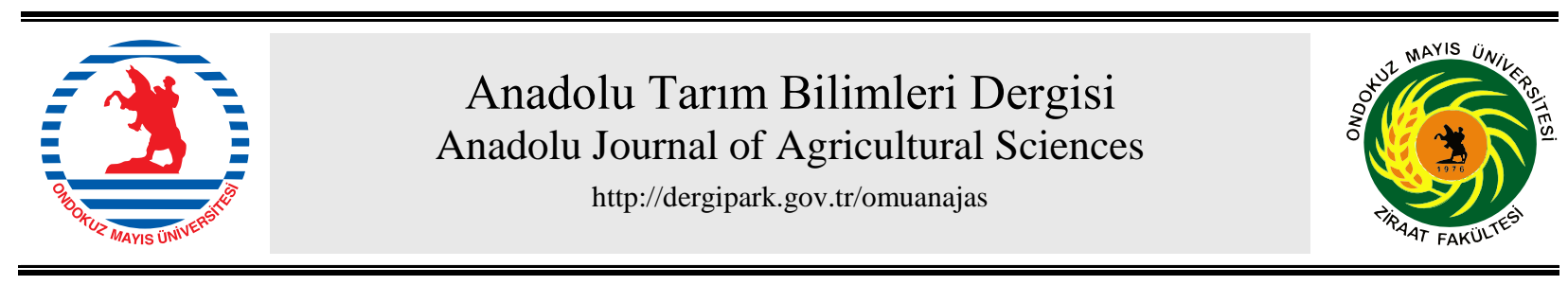

Araştırma/Research

\section{A deep learning based approach for the detection of diseases in pepper and potato leaves}

- Eser Sert ${ }^{\mathrm{a}^{*}}$
ISSN: 1308-8750 (Print) 1308-8769 (Online)

doi: 10.7161/omuanajas.805152

\begin{abstract}
${ }^{a}$ Malatya Turgut Özal Üniversitesi, Mühendislik ve Doğa Bilimleri Fakültesi, Bilgisayar Mühendisliği Bölümü, Malatya, Turkey
\end{abstract}
*Sorumlu yazar/corresponding author: eser.sert@ozal.edu.tr

Geliş/Received 05/10/2020 Kabul/Accepted 24/05/2021

\begin{abstract}
The present study proposes a Faster R-CNN Object Detection Approach with GoogLeNet Classifier (Faster R-CNN-GC) using image stitching, Faster R-CNN and GoogLeNet to detect pepper and potato leaves as well as leaf diseases in them. It is widely known that for a successful object detection performance, Faster R-CNN requires performing image labelling on a very high number of data, which will later train Faster R-CNN. However, this process is often very time-consuming. The present study mainly aims to shorten this process by designing an object detection approach which benefits from Faster R-CNN and GoogLeNet architecture. Firstly, Faster R-CNN and GoogLeNet were trained. Later, for the testing process, some of two-piece images were combined using an image stitching approach. Finally, using Faster R-CNN and GoogLeNet, pepper and potato leaves are detected and diseases are written on them. In addition, the proposed system was compared with Faster R-CNN Object Detection Approach with AlexNet Classifier (Faster R-CNN-AC), Faster R-CNN Object Detection Approach with SequezeNet Classifier (Faster R-CNN-SC) and Faster R-CNN. The findings of the experimental studies demonstrated that Faster R-CNN-GC displayed a higher object detection performance compared to other approaches.
\end{abstract}

Biber ve patates yapraklarındaki hastalıkların saptanması için derin öğrenme temelli bir yaklaşım

\section{ÖZET}

Bu çalışmada, görüntü birleștirme, daha hızlı-bölgesel evrişimsel sinir ağı (Faster R-CNN) ve GoogLeNet kullanılarak biber ve patates yapraklarını tespit eden ve tespit edilen yapraklardaki hastalık türünü gösteren, GoogLeNet sınıflandırıcılı Faster R-CNN nesne tespit yaklaşımı (Faster R-CNN-GC) önerilmiştir. Bilindiği gibi, Faster R-CNN'nin başarılı bir şekilde nesne tespitini gerçekleştirebilmesi için, çok fazla eğitim datası üzerinde imge etiketleme yapılması ve bu datalarla Faster R-CNN'nin eğitim sürecinden geçirilmesi gerekmektedir. Fakat bu süreç çok zaman alıcıdır. Bu çalışmadaki temel amaç bu süreci kısaltmak için Faster R-CNN ve GoogLeNet mimarisinin birlikte çalıştığı bir nesne tespit yaklaşımının tasarlanmasıdır. Çalışmada başlangıçta Faster R-CNN ve GoogLeNet'in eğitim süreci tamamlamıștır. Ardından test sürecine geçilmiș ve bazı test resimleri iki parçalı olduğu için görüntü birleştirme yaklaşımıyla bu görüntüler birleştirilmiştir. Ardından, Faster R-CNN ile resimdeki yaprak/yapraklar tespiti edilmiş ve GoogLeNet ile hastalık durumu belirlenmiştir. Bunlara ek olarak önerilen sistem, AlexNet sınıflandırıcılı Faster R-CNN nesne tespit yaklaşımı (Faster R-CNN-AC), SequezeNet sınıflandırıcılı Faster R-CNN nesne tespit yaklaşımı (Faster R-CNN-SC) ve Faster R-CNN ile karşılaştırılmıştır. Gerçekleştirilen deneysel çalışmalar önerilen Faster R-CNN-GC'nin diğer yaklaşımlara göre daha üstün bir şekilde nesne tespitini gerçekleştirdiği göstermiştir.
Keywords: Leaf Disease Detection Faster R-CNN Object Detection GoogLeNet SequezeNet AlexNet

Anahtar Sözcükler: Yaprak hastalık tespiti Faster R-CNN Nesne tespiti GoogLeNet SequezeNet AlexNet 


\section{Introduction}

The detection of leaf diseases using image detection approaches is one of the most popular research topics in the field of agriculture today. Because most plant diseases usually display symptoms in a visible spectrum, they paved the way for the effective use of various tools such as artificial intelligence, image processing and computer vision systems. These tools play a critical role in the automatic detection of plant diseases and determination of disease type, which eventually increases agricultural productivity. Various approaches employed in this field are logistic regression, K-nearest neighbors (K-NN), convolutional neural network (CNN), support vector machine (SVM) and decision tree (Geetharamani and Pandian, 2019). However, the number of studies benefiting from CNN and Faster R-CNN is fairly limited.

Proposed by Yann LeCun in 1988, CNN has been developed since then. In recent years, thanks to a gradual development in the number of layers in its architecture, CNN performances have remarkably improved, and different CNN architectures have so far been proposed. Thus, it became possible to reach high accuracy rates in different applications such as image classification, segmentation and object detection. AlexNet, GoogLeNet Inception V3, Inception V4, VGG net, DenseNets, SqueezeNet and Microsoft ResNet can be listed as major CNN architectures (Too et al., 2019). Faster R-CNN is an important object detection approach proposed by Ren et al. (2015). As it performs object detection with a considerably high performance, it has been used in a number of quite different applications in recent years. Both Faster R-CNN and CNN are deep neural networks which have displayed an effective performance in various applications such as object detection, object classification and speech recognition. As a result of the literature studies, it was seen that CNN was used for the following purposes:

- Biological image classification (Qin et. al, 2020)

- Brain tumor detection (Özyurt et al., 2019; Özyurt et al., 2020) and diagnosis system (Sert et al., 2019)

- Chinese text recognition (Wang and Du, 2021)

- Facial expression recognizer (Teja et al., 2020)

- Finger vein recognition (Zhao et al., 2020)

- Industrial food packaging (Medus et al., 2021)

- Leaf disease classification (Deeba and Amutha, 2020)

- Speech emotion recognition (Kwon, 2020)

- Underwater target detection (Zeng et al., 2021)

In the present study, Faster R-CNN Object Detection Approach with GoogLeNet Classifier (Faster R-CNN-GC) approach, which combined Faster R-CNN and GoogLeNet, was proposed in order to detect pepper and potato leaves and diseases. Some images were combined using an image stitching (Brown and Lowe, 2007) approach because they consisted of two pieces, which helped perform image processing on some leaf images from a wider angle. In this study, using GoogLeNet as a classifier, it is aimed to maximize the prediction performance of Faster R-CNN.

One of the studies in which deep learning was used in the agricultural field was conducted by Zhou et al. (2019). This study proposed a K-Means clustering algorithm (FCM-KM algorithm) and Faster R-CNN fusion-based approach in order to detect rice leaf diseases and reported that three different types of leaf diseases, namely rice blast, bacterial blight and sheath blight, were detected with an average accuracy rate of 97.2\%. Sibiya and Sumbwanyambe (2019) proposed a CNN-based approach for the detection and classification of plant diseases. Hu et al. (2019) proposed a deep CNN-based approach in order to detect tea leaf diseases, which yielded an average accuracy rate of 92.5\%. Özguven et al. (2019) proposed a Faster R-CNN-based approach for the automatic detection of sugar beet leaf diseases (Cercospora beticola Sacc.) and achieved an accuracy rate of 95.48\%. Waheed et al. (2020) proposed a CNN-based approach for corn leaf disease recognition and classification. Karlekar and Seal (2020) proposed a CNN-based approach for soybean plant diseases recognition.

The innovative contributions of the present study are summarized below:

1) Image stitching and CNN have not been used together to detect leaf diseases in previous studies in the existing literature.

2) In order to predict the object accurately using Faster R-CNN, it is necessary to train Faster R-CNN using training data which were subjected to a number of image labelling processes. A low number and quality of image labelling in these processes result in a poor object detection performance. In the present study, Faster R-CNN and GoogLeNet were used together in order to offer a solution to this problem.

3) The proposed approach used a lower number of training data lower than that of a conventional Faster R-CNN training process, which shortened the image labelling process to a great extent.

4) In order to measure the performance of the proposed approach, Faster R-CNN-GC approach was compared Faster R-CNN Object Detection Approach with AlexNet Classifier (Faster R-CNN-AC), Faster R-CNN Object Detection Approach with SequezeNet Classifier (Faster R-CNN-SC) and Faster R-CNN. It was reported that the proposed approach displayed a much higher accuracy rate compared to other approaches. 
Dataset organization and image stitching, CNN, Faster R-CNN, the proposed approach and other approaches, Faster R-CNN approach, performance measurement metrics are described in Section 2. Experimental studies are presented in Section 3. Section 4 concludes the present study.

\section{Material and Methods}

\subsection{Dataset}

The present study benefited from Plant Village dataset (Hughes and Salathé, 2015) and leaf images captured from pepper and potato fields in Malatya by the author in order to create a training dataset for Faster R-CNN. Honor 8X mobile phone was used for photograph capturing. As a result, a total of 544 pepper and potato leaf images were obtained using these captured photographs and Plant Village dataset images. These leaf images consisted of five different categories, namely Potato Late Blight, Potato Healthy, Potato Early Blight, Pepper Bell Healthy and Pepper Bell Bacterial Spot. Image augmentation was applied to these 544 images to improve the performance of Faster RCNN. 90-degree image rotation, contrast enhancing and brightness enhancing were applied to leaf images in Step 1, 2 and 3 of this process respectively. Thus, a dataset containing 4 x $544=2176$ leaf images was created. The numbers of leaves from Pepper Bell Bacterial Spot, Pepper Bell Healthy, Potato Early Blight, Potato Healthy and Potato Late Blight species in the database are 460, 671, 448, 115 and 482, respectively. This dataset created within the scope of the study is named as Dataset 1. Figure 1-a shows some of images from Dataset 1.

Another dataset containing 6023 pepper and potato leaf images was created using the photographs taken by the author from the pepper and potato fields in Malatya and the images from the Plant Village data set. This dataset as named Dataset 2 was used for the training process of AlexNet, GoogleNet and SequeezeNet and the testing process of all approaches. 4627 images from Dataset 2 were included in the training and validation process of AlexNet, GoogleNet and SequeezeNet. 3239 images (70\%) were used for the training process, while 1388 images (30\%) were used for the validation process. Number of Pepper Bell Bacterial Spot, Pepper Bell Healthy, Potato Early Blight, Potato Healthy and Potato Late Blight types leaves used for the training process are 698, 1035, 700, 106 and 700, respectively. Numbers of Pepper Bell Bacterial Spot, Pepper Bell Healthy, Potato Early Blight, Potato Healthy and Potato Late Blight types leaves used for the validation process are 299, 443, 300, 46 and 300, respectively. The remaining $6048-4628=1420$ images were used for the testing process of all approaches. Leaf numbers of Pepper Bell Bacterial Spot, Pepper Bell Healthy, Potato Early Blight, Potato Healthy and Potato Late Blight types used for the testing process are 310, 447, 312, 41 and 310, respectively. Figure 1-b shows some images from Dataset 2.

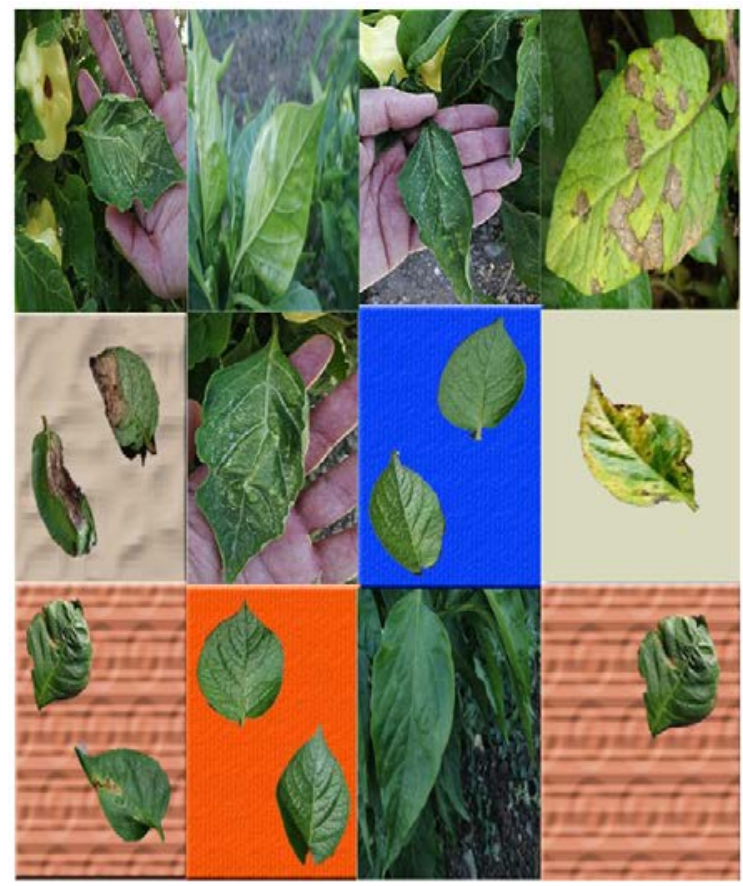

a

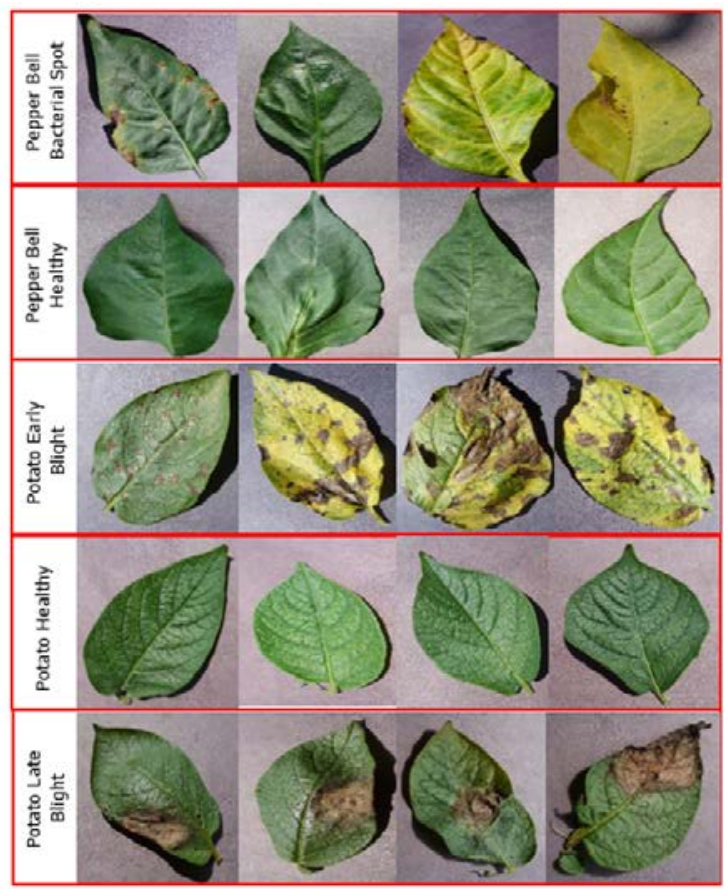

b

Figure 1. a) Some of images from Dataset 1, b) Some of images from Dataset 2.

Şekil 1. a) Dataset 1'den bazı görüntüler, b) Dataset 2' den bazı görüntüler. 


\subsection{Image stitching}

Image stitching enables combining multiple images captured from more than one camera or images in different pieces by applying a few steps to overlapping fields of those images in order to produce panoramic or high resolution images. The present study employs an image stitching approach to combine different leaf images. The experimental studies in this study demonstrated that the approach proposed by Brown and Lowe (2007) displayed the highest image stitching performance. Therefore, this approach was used to combine images in different segments in the testing process. Brown and Lowe (2007) proposed a successful approach for a fully automatic panoramic stitching. This study employed invariant local features and a probabilistic model in order to verify image matches. Thus, it made it possible to detect several panoramic images in unordered image sets, and these image sets were combined automatically without any user login. More detailed information about this study can be found in (Brown and Lowe, 2007).

\section{3. $C N N$}

CNN is a type of multilayer perceptron (MLP). It is a feedforward neural network which was inspired by human brain (Lumini and Nanni, 2019). It has been widely used in various different fields such as medicine, security and satellite images for the purposes of classification and segmentation due to its successful performance. It contains various layers in its structure such as convolution, activation, pool, fully-connected and classification. It is possible to improve CNN performance by connecting these layers in different combinations and numbers (Lumini and Nanni, 2019).

\subsection{The proposed approach}

The proposed Faster R-CNN-GC approach benefits from Faster R-CNN in order to detect pepper and potato leaves in the image set and GoogLeNet classifier in order to detect types of diseases and match the disease with the related leaf. Faster R-CNN-GC, which is a comparison approach, differs from Faster R-CNN-AC in that it employs GoogLeNet as a classifier, whereas Faster R-CNN-SC employs SequezeNet as a classifier. The functions of these three approaches are summarized in Figure 2. The proposed approach consists of seven steps:

\section{Training Process}

Step -1: Data labelling process of leaf images in the dataset

Step -2: Faster R-CNN training

Step-3: Training and validation processes of AlexNet, SequezeNet and GoogleNet architectures

\section{Testing Process}

Step-4: Object detection on images using Faster R-CNN

Step-5: Segmentation of object detected

Step-6: Detection of leaf diseases using AlexNet, SequezeNet and GoogleNet classifiers

Step-7: Writing disease names on leaf images

\subsubsection{Training processes}

- Step-1: Data labelling process of leaf images in the dataset: As described in detail in Section "2. 1. Dataset”, 2176 training images from Dataset 1 were used for Faster R-CNN. These images are labelled in Step 1 prior to the training process. In this process, all leaves in the image dataset were labelled as "leaf".

- Step-2: Faster R-CNN training: In Step-2, 2176 labelled images were used to perform Faster R-CNN training.

- Step-3: Training and validation processes of AlexNet, SequezeNet and GoogleNet architectures: As described in detail in Section "2. 1. Dataset”, a dataset containing 3239 training and 1388 validation images from Dataset 2 was prepared. This dataset was used in Step-3 to complete the training processes of AlexNet, SequezeNet and GoogleNet architectures.

\subsubsection{Testing processes}

- Step-4: Object detection on images using Faster R-CNN: In this step, the leaves in the Image, which is the input image, are detected using Faster R-CNN. The detected leaves are seen in the Faster_R-CNN_image seen in Figure 7. 
As described in detail in Section "2.1. Dataset”, 1420 test images from Dataset 2 were used the testing process. Because 50 out of 1420 testing images were two-piece images.

- Step-5: Segmentation of object detected: The segmentation of the leaves detected in the previous step is carried out in this step. Since each leaf in the Image is framed by Faster R-CNN, segmentation of these leaves was performed using these frame coordinates. As can be seen in Figure 2, since 7 leaf images are detected in the Image, these 7 leaf images are segmented.

- Step-6: Detection of leaf diseases using AlexNet, SequezeNet and GoogleNet classifiers: In the first step, the disease types of the segmented leaves were estimated using the Faster R-CNN-GC approach, which includes GoogLeNet classifier. In the second step, the disease types of the segmented leaves were estimated using the Faster R-CNN-AC approach, which includes AlexNet Classifier. In the third step, the disease types of the segmented leaves were determined with the Faster R-CNN-SC approach, which includes the SequezeNet Classifier. At the output of AlexNet, SequezeNet and GoogleNet classifiers for each segmented leaf image, only one of the disease types 'Pepper Bell Bacterial Spot, Pepper Bell Healthy, Potato Early Blight, Potato Healthy and Potato Late Blight' is obtained as a class output.

- Step-7: Writing disease names on leaf images: In this step, the classification results obtained through the AlexNet, SequezeNet and GoogleNet classifiers in the previous step are written on the Image.

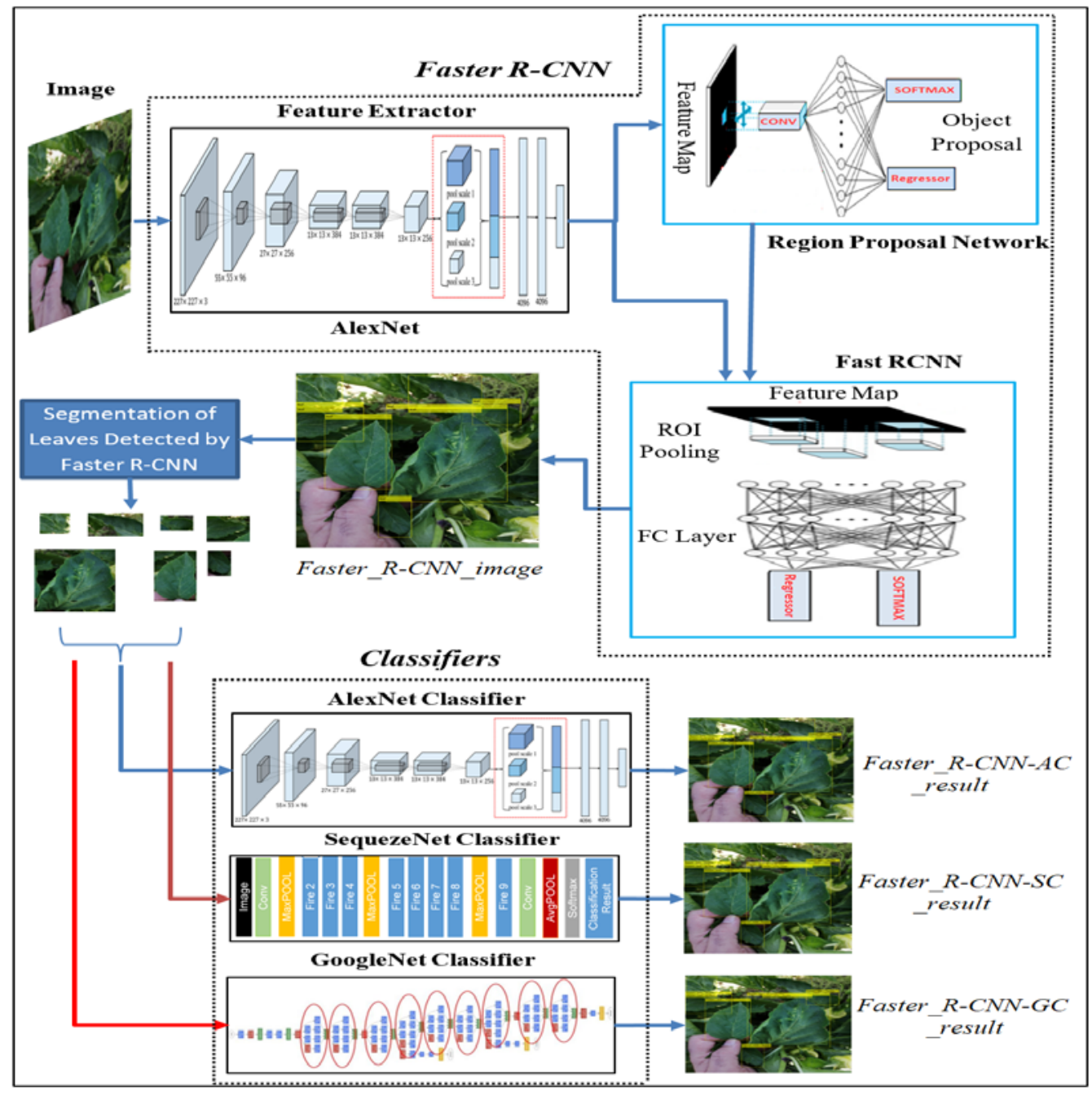

Figure 2. Diagram describing the working structure of Faster R-CNN-GC, Faster R-CNN-AC and Faster R-CNN-SC. Şekil 2. Faster R-CNN-GC, Faster R-CNN-AC ve Faster R-CNN-SC'nin çalışma yapısını açıklayan diyagram. 


\subsection{Faster R-CNN detection approach}

An approach which could detect five different types of leaves, namely Pepper Bell Bacterial Spot, Pepper Bell Healthy, Potato Early Blight, Potato Healthy ve Potato Late_Blight, was designed using only Faster R-CNN in order to compare the proposed Faster R-CNN-AC approach with other approaches. The structure of a Faster R-CNN which is used to detect leaf diseases is shown in Figure 3. First, the training process of Faster R-CNN was completed. As described in detail in Section "2. 1. Dataset”, 2176 images in Dataset 1 were used for Faster R-CNN training. These images were labelled as Potato Late Blight, Potato Healthy, Potato Early Blight, Pepper Bell Healthy and Pepper Bell Bacterial Spot. Later, this dataset was used to perform the training of Faster R-CNN. As described in detail in Section “2. 1. Dataset”, 1420 test images in Dataset 2 were used the testing process of Faster R-CNN. The object detection result obtained with this approach is named as Faster_R-CNN_result.

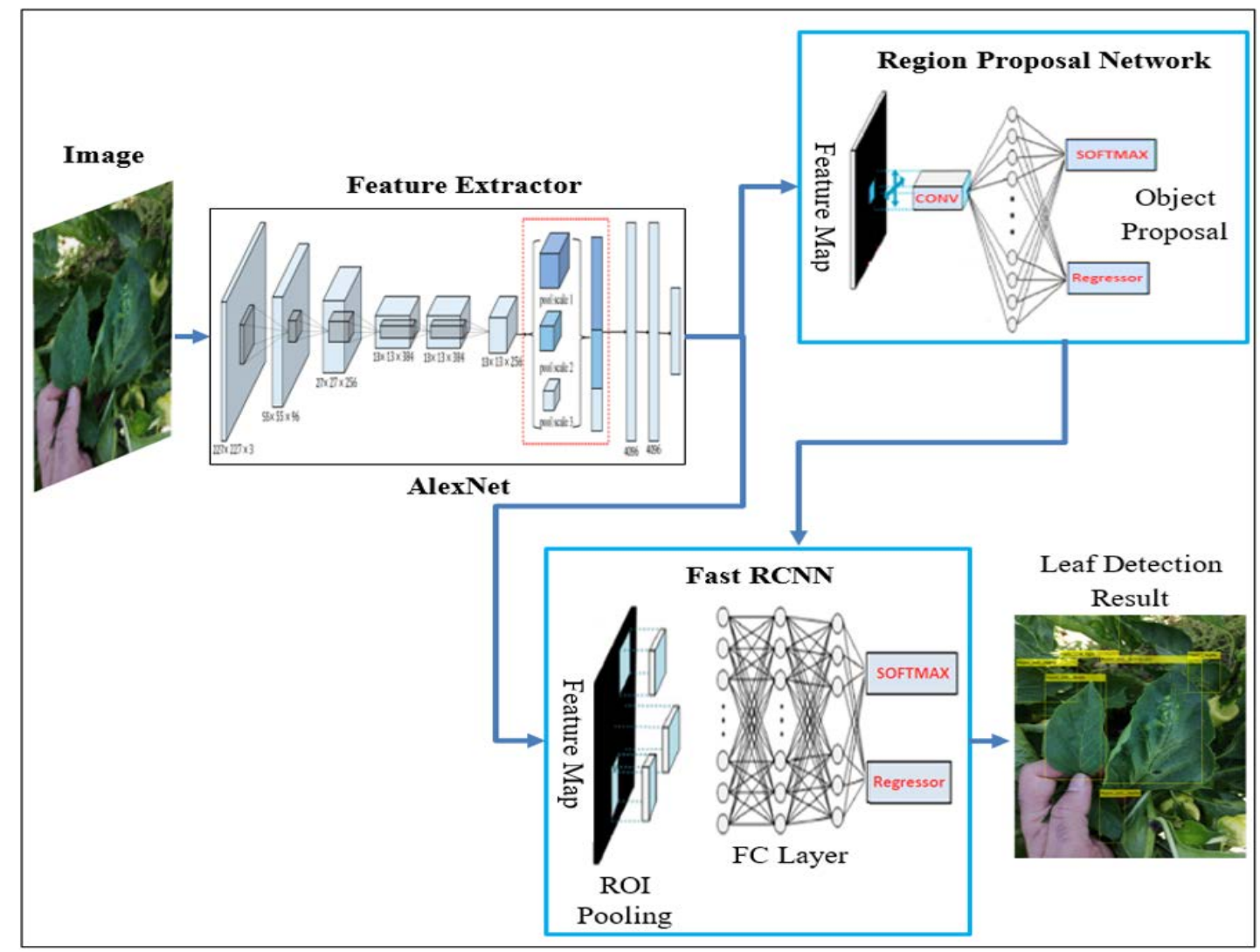

Figure 3. Architecture of Faster R-CNN.

Şekil 3. Faster R-CNN’nin Mimarisi.

\subsection{Performance measurement metrics}

Accuracy, F1-score, precision and recall were used to compare the performances of CNN-based approaches in the present study (Sokolova and Lapalme, 2009). Accuracy: It is one of the most popular criteria used for the assessment of a CNN model. A higher accuracy value points to a CNN model with a higher level of performance. Accuracy equation is defined as follows:

Accuracy $=\frac{T P+T N}{T P+T N+F P+F N}$

Here, $\mathrm{TP}=$ True Positive, $\mathrm{TN}=$ True Negative, $\mathrm{FP}=$ False Positive and FN = False Negative.

Precision and recall used to compare CNN-based approaches in the present study are defined as follows: 
Precision $=\frac{T P}{T P+F P}$

Recall $=\frac{T P}{T P+F N}$

F1-score: It is a popular and highly reliable method to compare different machine learning approaches. F1-score corresponds to the harmonic mean of precision and recall values and is defined as follows:

F1-score $=\frac{2 * \text { Precision } * \text { Recall }}{\text { Precision }+ \text { Recall }}$

\section{Results and Discussion}

- Experiment - 1: Faster R-CNN Training

Training accuracy and loss curves obtained from the training process of Faster R-CNN in Faster R-CNN-AC, Faster R-CNN-SC and Faster R-CNN-GC approaches using a dataset of 2176 'leaf' labelled images are shown in Figure 4.
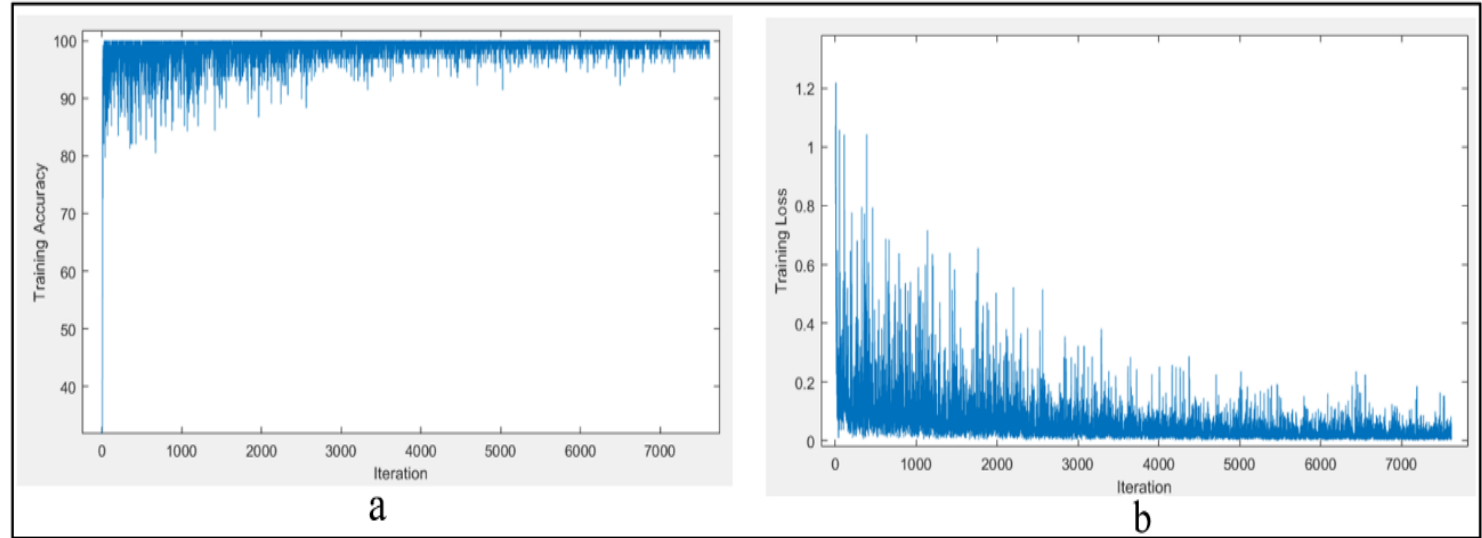

Figure 4. a) Plot for training accuracy, b) Plot for training loss.

Şekil 4. a) Ĕ̌itim doğruluğu grafiği, b)Ĕ̌itim kaybı grafiği.

- Experiment - 2: CNN Models Training

In this experimental process, training and validation processes of AlexNet, GoogleNet and SequeezeNet based classifiers in Faster R-CNN-AC, Faster R-CNN-SC and Faster R-CNN-GC were performed. Accuracy and loss curves obtained from 3239 training and 1388 validation images for GoogLeNet based classifier are shown in Figure 5.

- Experiment - 3: All approaches were tested in this experiment process. As a result, confusion matrices in Figure 6 and detailed comparison results given in Table 1-2 were obtained from 1395 images used to compare all three approaches. Results of four metrics for the four approaches are presented in Table 2. It can be understood the table that all performance comparison values of Faster R-CNN-GC were higher compared to other approaches. These findings demonstrate that Faster R-CNN-GC performed the detection of leaf diseases with a higher level of accuracy compared to other four approaches. 


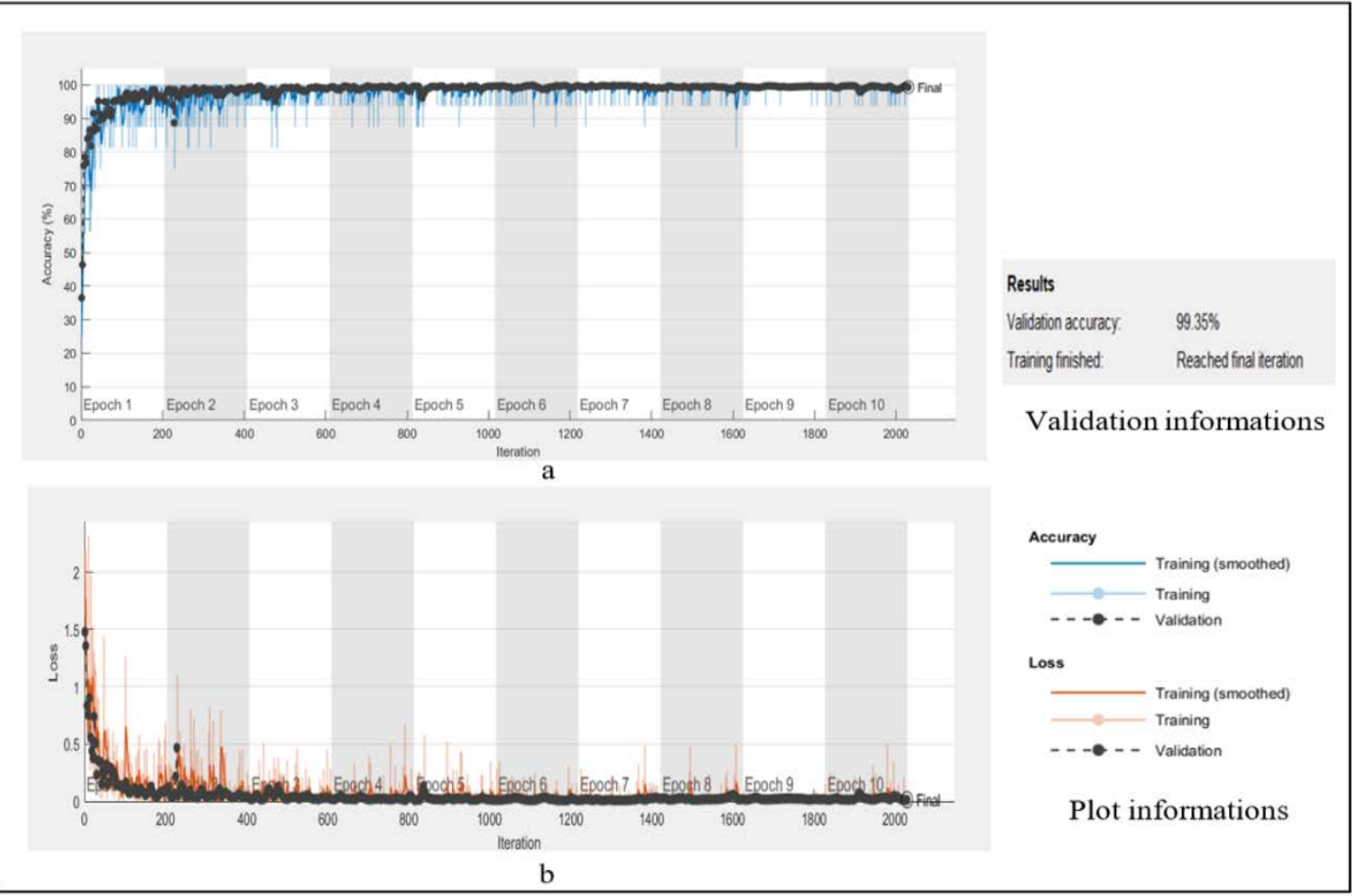

Figure 5. a) Plot for accuracy of GoogLeNet, b) Plot for loss of GoogLeNet.

Şekil 5. a) GoogLeNet 'in doğruluk grafiği b) GoogLeNet' in kayıp grafiği.

Table 1. Detailed efficiency metrics for Faster R-CNN-GC

Çizelge 1. Faster $R-C N N-G C$ için ayrıntıll verimlilik metrikleri

\begin{tabular}{|c|c|c|c|c|c|}
\hline Class & Accuracy (\%) & Precision & Recall & F1 Score & $\begin{array}{c}\text { Overall } \\
\text { Accuracy }\end{array}$ \\
\hline $\begin{array}{l}\text { Pepper Bell Bacterial } \\
\text { Spot }\end{array}$ & 99.35 & 0.99 & 0.98 & 0.98 & \multirow{5}{*}{98.06} \\
\hline Pepper Bell Healthy & 99.21 & 0.98 & 0.99 & 0.99 & \\
\hline Potato Early Blight & 99.5 & 0.99 & 0.99 & 0.99 & \\
\hline Potato Late_Blight & 98.92 & 0.99 & 0.96 & 0.98 & \\
\hline \multirow[t]{3}{*}{ Potato Healthy } & 99.14 & 0.83 & 0.90 & 0.86 & \\
\hline & & $\begin{array}{l}\text { Weighted- } \\
\text { Precision }\end{array}$ & $\begin{array}{l}\text { Weighted- } \\
\text { Recall } \\
\end{array}$ & $\begin{array}{l}\text { Weighted- F1 } \\
\text { Score } \\
\end{array}$ & \\
\hline & & 0,98 & 0.98 & 0.98 & \\
\hline
\end{tabular}

Table 2. Comparing the performance of the four approaches with metrics Çizelge 2. Dört yaklaşımın performansının metriklerle karşılaşıtırması

\begin{tabular}{lcccc}
\hline & Overall Accuracy (\%) & Weighted- Precision & Weighted- Recall & Weighted-F1 Score \\
\hline Faster R-CNN-GC & 98.06 & 0,98 & 0.98 & 0.98 \\
\hline Faster R-CNN-AC & 96.7 & 0.97 & 0.97 & 0.97 \\
\hline Faster R-CNN-SC & 95.41 & 0.95 & 0.95 & 0.95 \\
\hline Faster R-CNN & 82.58 & 0.83 & 0.84 & 0.83 \\
\hline
\end{tabular}




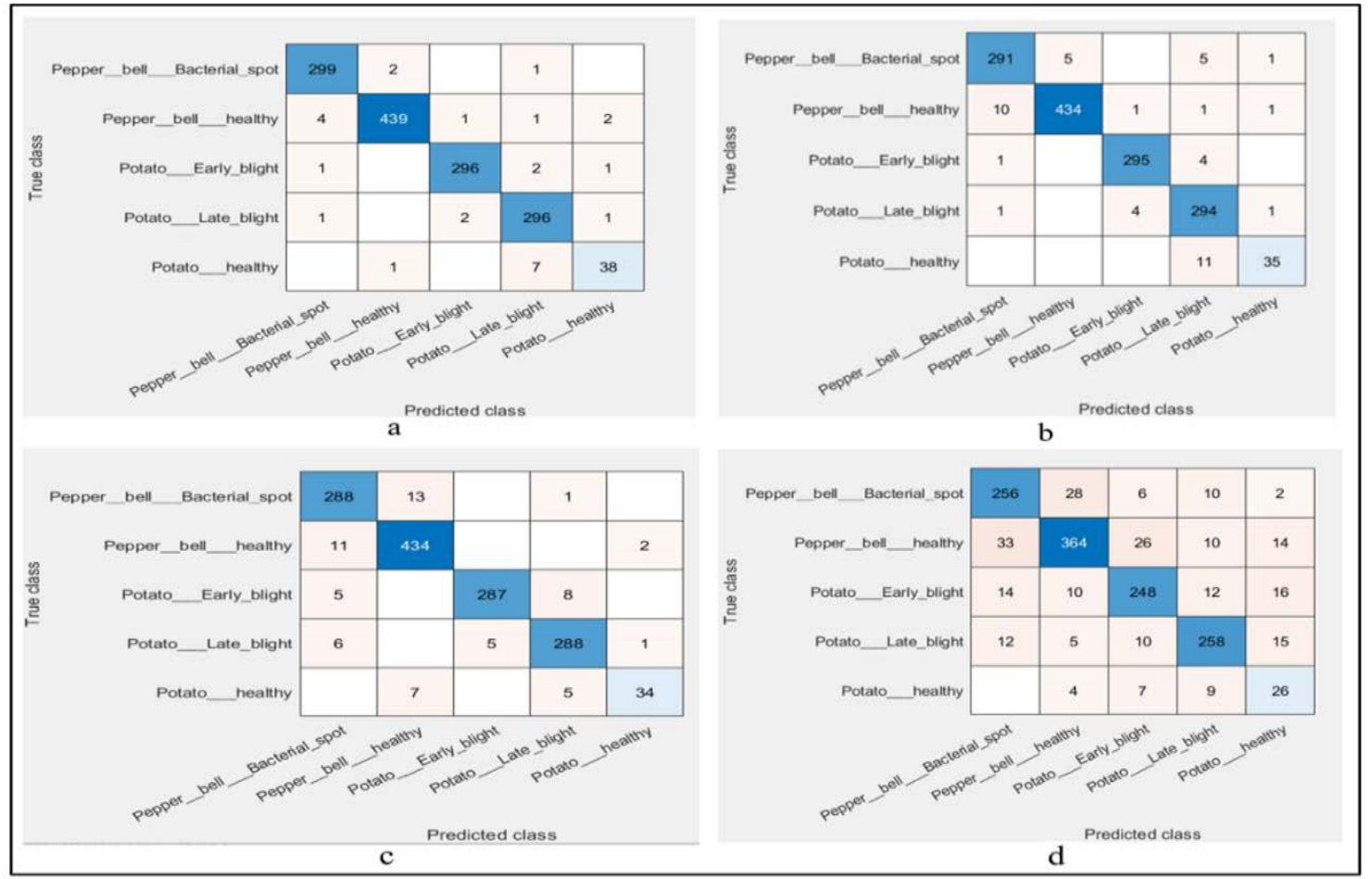

Figure 6. a) Confusion matrix of Faster R-CNN-GC, b) confusion matrix of Faster R-CNN-AC, c) confusion matrix of Faster R-CNN-SC, d) confusion matrix of Faster R-CNN.

Şekil 6.a) Faster R-CNN-GC'nin karmaşıklık matrisi, b) Faster R-CNN-AC’nin karmaşıklık matrisi, c) Faster R-CNNSC'nin karmaşıklık matrisi, d) Faster R-CNN'nin karmaşılklı matrisi.

- Experiment - 4: In the present study, 50 testing images were combined using image stitching, as shown in Figure 7-a and Figure 7-b. Figure 7-c was obtained after image stitching was applied to these images. These figures demonstrated that the related images were combined with a high level of image quality.

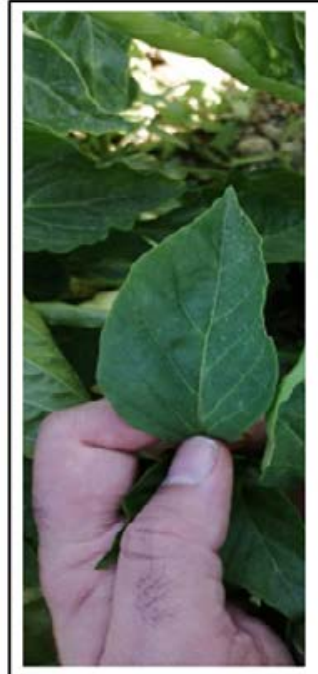

a

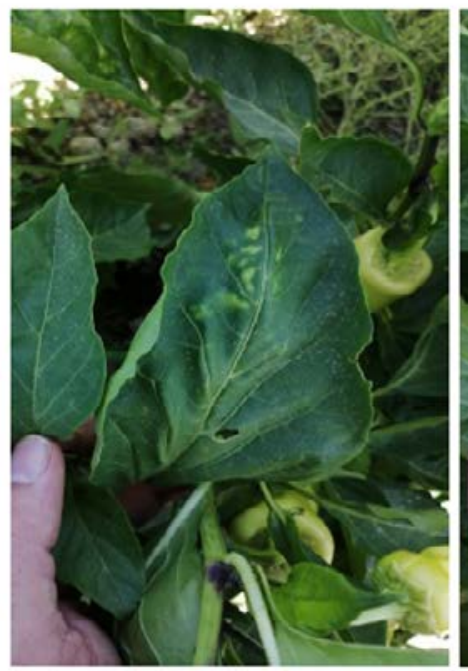

b

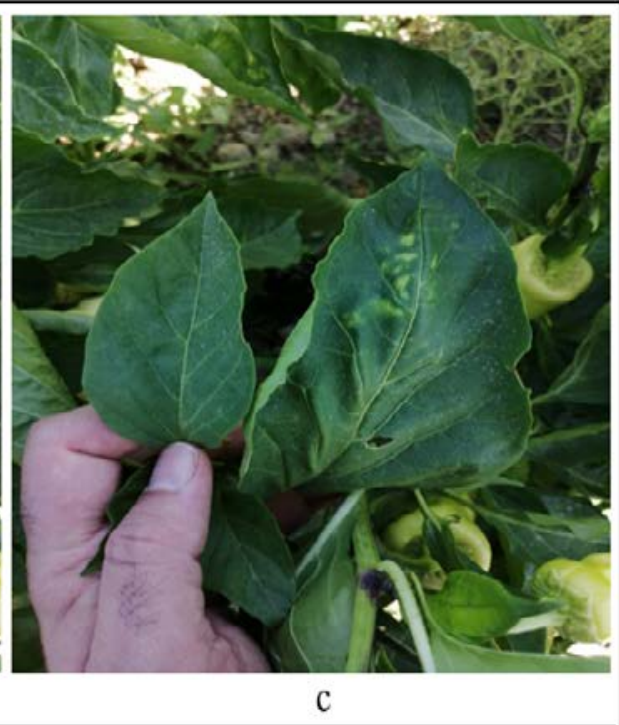

$\mathrm{C}$

Figure 7. a) image 1, b) image 2, c) img (the result image with image stitching applied).

Şekil 7.a) image 1, b) image 2, c) img (görüntü birleştirme uygulanmış sonuç görüntüsü).

- Experiment - 5: The output images obtained from a sample input image shown in Figure 8-a are shown in Figure 8-b and Figure 8-c in order to measure higher performance of the proposed Faster R-CNN-GC approach. 
Faster_R-CNN_image, which is the result image of Faster R-CNN used in the Faster R-CNN-GC, is shown in Figure 8-b. It can be observed that the detected pepper leaves were labelled as 'leaf'. cropped_images corresponding to the segmented images are shown in Figure 8-c. The object detection results obtained using four different approaches are shown in Figure 9. The ground truth labels of the leaves detected in Figure 8-b are Pepper Bell Healthy, Pepper Bell Healthy, Pepper Bell Healthy, Pepper Bell Healthy, Pepper Bell Healthy, Pepper Bell Bacterial Spot and Pepper Bell Healthy respectively. These ground truth labels were correctly detected by Faster R-CNN-GC. But Faster R-CNNAC and Faster R-CNN-SC misclassified only one of the detected leaves. Faster R-CNN could not detect two leaves and misclassified one leaf. It can be stated that Faster R-CNN-GC displayed a performance with an accuracy rate of 100\%, while Faster R-CNN-AC, Faster R-CNN-SC and Faster R-CNN displayed an accuracy rate of 85,71\%, 85,71\% and $57,14 \%$ respectively.

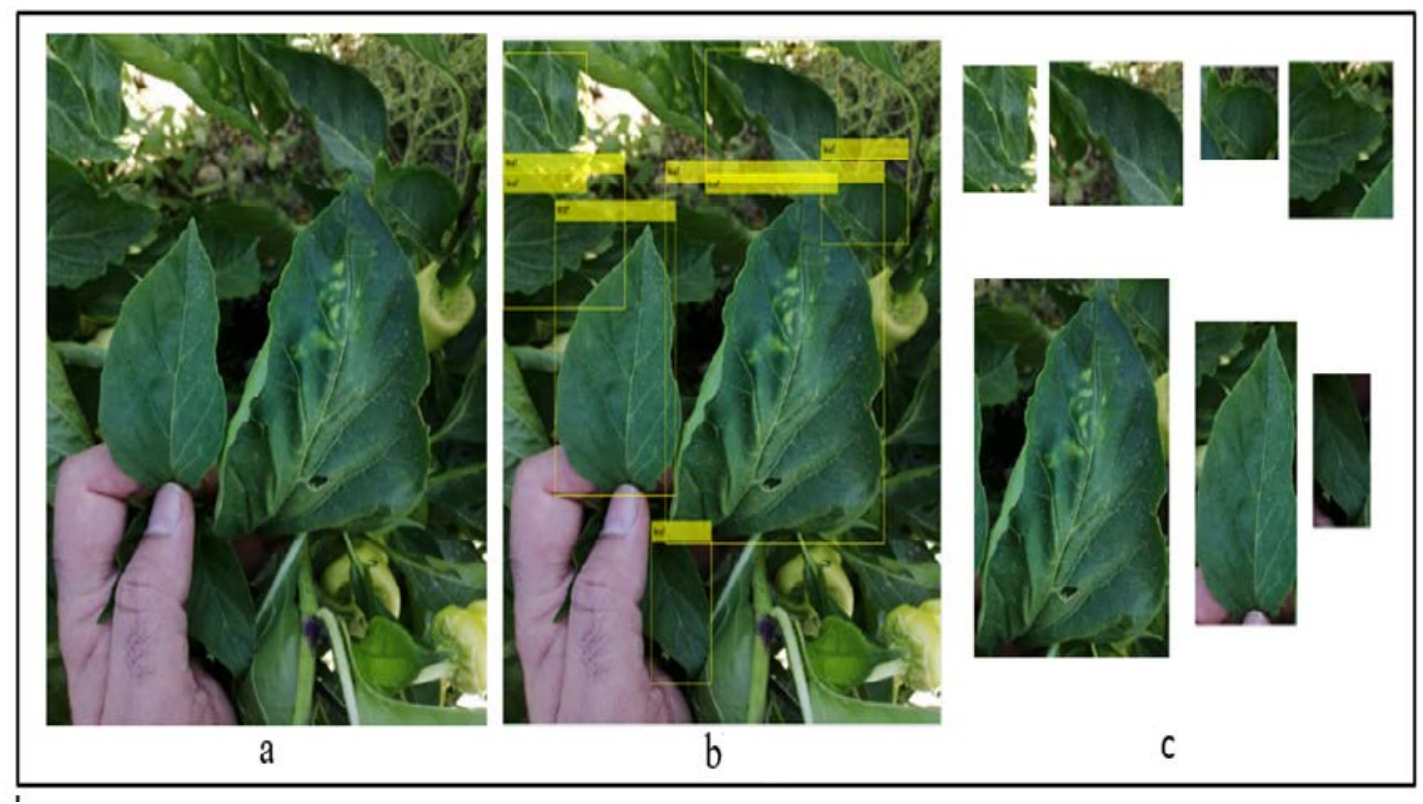

Figure 8. a) img, b) Faster_R-CNN_image, c) cropped_image.

Şekil 8. a) img, b) Faster_R-CNN_image, c) cropped_image.

Faster R-CNN training in Experiment -1 and CNN models training processes in Experiment -2 were been successfully completed. As can be seen from the results in Table-1 and Table 2, the Faster R-CNN-GC approach detected diseases in leaves more successfully than other approaches. At the same time, the results in these tables show that the GoogLeNet classifier used in the Faster R-CNN-GC approach more successfully classifies the diseases in pepper and potato leaves. The visual analysis results performed on Figure 8 in Experiment -5 show again the superiority of the proposed approach.

As given in Table 3, the performance of the proposed approach was compared with other CNN-based plant disease detection and classification approaches in the existing literature in terms of their respective accuracy rates. It can be observed that the proposed approach had a higher accuracy rate compared to other approaches.

Table 3. Comparative of the proposed approach and other approaches in the literature Çizelge 3. Önerilen yaklaşımla literatürdeki diğer yaklaşımların karşılaştırılması

\begin{tabular}{ccccc}
\hline Authors & Year & Number of Class and Plant & Methods & Accuracy (\%) \\
\hline Proposed Approach & 2020 & 5 class for 2 type plant & Faster R-CNN and GoogLeNet & 98.06 \\
\hline Zhou et al (2019) & 2019 & 3 class for 1 type plant & FCM-KM algorithm and Faster R-CNN fusion & 97.2 \\
\hline Ozguven et al (2019) & 2019 & 4 class for 1 type plant & Faster R-CNN & 95.48 \\
\hline Sibiya et al. (2019) & 2019 & 4 class for 1 type plant & CNN & 92.85 \\
& & & CIFAR10-quick (CNN) & 92.5 \\
\hline Hu et al (2019) & 2019 & 4 class for 1 type plant & & \\
\hline
\end{tabular}




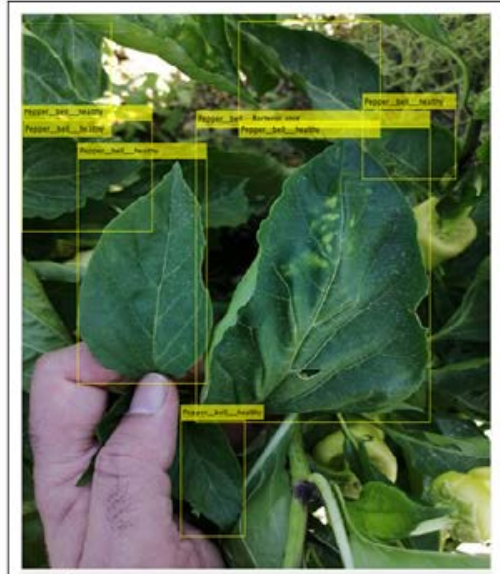

a

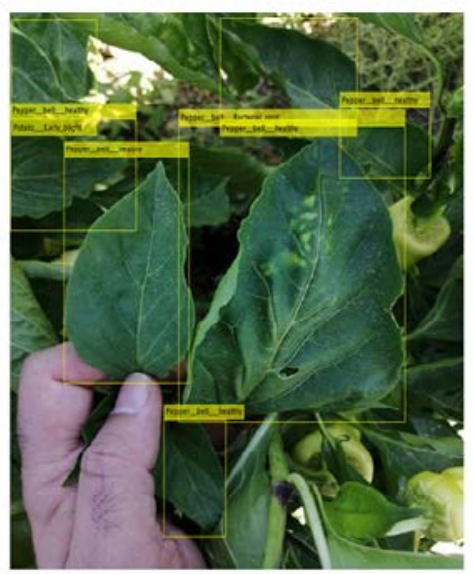

b

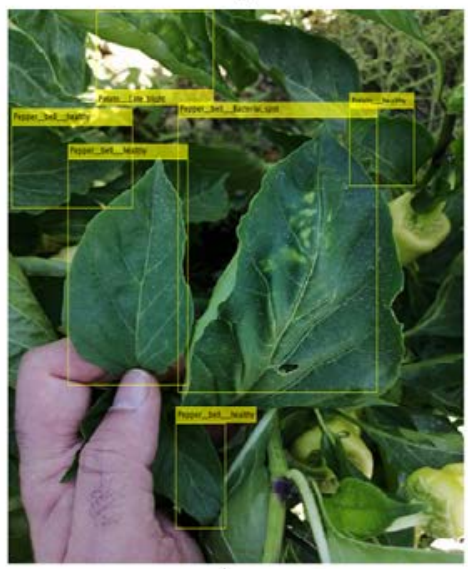

d

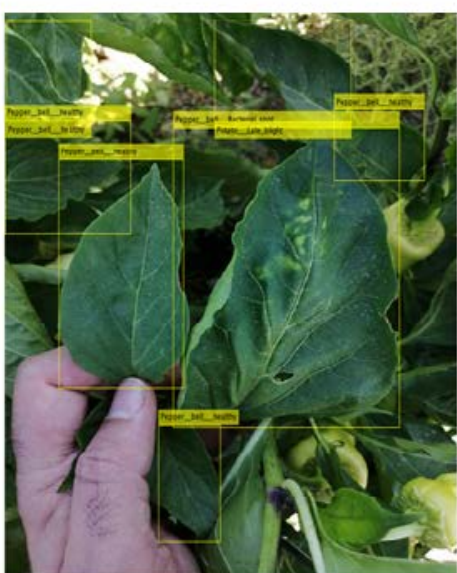

c

Figure 9. a) Faster_R-CNN-GC_result, b) Faster_R-CNN-AC_result, c) Faster_R-CNN-SC_result, d) Faster_RCNN_result.

Şekil 9. a) Faster_R-CNN-GC_result, b) Faster_R-CNN-AC_result, c) Faster_R-CNN-SC_result, d) Faster_RCNN_result.

\section{Conclusion}

In the present study, Faster R-CNN and GoogLeNet were used together in the proposed Faster R-CNN-GC approach in order to reduce the image labeling process required for the training process of Faster R-CNN. Two-piece images were combined using an image stitching approach in the testing process and thus a more effective object detection was performed thanks to the enlarged images. The proposed Faster R-CNN-GC was compared with Faster R-CNN-SC and Faster R-CNN. The experimental studies clearly demonstrated that the proposed Faster R-CNN-GC approach displayed a higher performance of leaf disease detection compared to other approaches. In conclusion, it can be argued that an innovative and highly efficient object detection approach that can be used in the agricultural field has been introduced to the existing literature.

\section{References}

Brown, M., Lowe D.G., 2007. Automatic panoramic image stitching using invariant features. International Journal of Computer Vision, 74(1):59-73. doi:10.1007/s11263-006-0002-3.

Deeba, K., Amutha, B., 2020. ResNet-deep neural network architecture for leaf disease classification. Microprocessors and Microsystems, 103364 (In Press). doi:10.1016/j.micpro.2020.103364.

Geetharamani, G., Pandian, A., 2019. Identification of plant leaf diseases using a nine-layer deep convolutional neural network. Computers \& Electrical Engineering, 76: 323-338. doi:10.1016/j.compeleceng.2019.04.011. 
Hu, G., Yang, X., Zhang, Y., Wan, M., 2019. Identification of tea leaf diseases by using an improved deep convolutional neural network. Sustainable Computing: Informatics and Systems, 24:100353. doi:10.1016/j.suscom.2019.100353.

Hughes, D., Salathé, M., 2015. An open access repository of images on plant health to enable the development of mobile disease diagnostics. arXiv Preprint arXiv:1511.08060.

Karlekar, A., Seal, A., 2020. SoyNet: Soybean leaf diseases classification. Computers and Electronics in Agriculture, 172:105342. doi:10.1016/j.compag.2020.105342

Kwon, S., 2020. MLT-DNet: Speech emotion recognition using 1D dilated CNN based on multi-learning trick approach. Expert Systems with Applications, 167:114177. doi:10.1016/j.eswa.2020.114177.

Lumini, A., Nanni, L., 2019. Deep learning and transfer learning features for plankton classification. Ecological informatics, 51: 33-43. doi:10.1016/j.ecoinf.2019.02.007.

Medus, L. D., Saban, M., Francés-Víllora, J. V., Bataller-Mompeán, M., Rosado-Muñoz, A., 2021. Hyperspectral image classification using CNN: application to industrial food packaging. Food Control, 125:107962. doi:10.1016/j.foodcont.2021.107962.

Ozguven, M. M., Adem, K., 2019. Automatic detection and classification of leaf spot disease in sugar beet using deep learning algorithms. Physica A: Statistical Mechanics and its Applications, 535: 122537. doi:10.1016/j.physa.2019.122537.

Özyurt, F., Sert, E., Avci, E., Dogantekin, E., 2019. Brain tumor detection based on Convolutional Neural Network with neutrosophic expert maximum fuzzy sure entropy. Measurement, 147:106830. doi:10.1016/j.measurement.2019.07.058.

Özyurt, F., Sert, E., Avc1, D., 2020. An expert system for brain tumor detection: Fuzzy C-means with super resolution and convolutional neural network with extreme learning machine. Medical hypotheses, 134:109433. doi:10.1016/j.mehy.2019.109433.

Qin, J., Pan, W., Xiang, X., Tan, Y., Hou, G., 2020. A biological image classification method based on improved CNN. Ecological Informatics, 58:101093. doi:10.1016/j.ecoinf.2020.101093.

Ren, S., He, K., Girshick, R., Sun, J. (2015). Faster R-CNN: Towards real-time object detection with region proposal networks. In Advances in Neural Information Processing Systems, 28: 91-99.

Sert, E., Özyurt, F., Doğantekin, A., 2019. A new approach for brain tumor diagnosis system: Single image super resolution based maximum fuzzy entropy segmentation and convolutional neural network. Medical hypotheses, 133:109413. doi:10.1016/j.mehy.2019.109413.

Sibiya, M., Sumbwanyambe, M., 2019. A computational procedure for the recognition and classification of maize leaf diseases out of healthy leaves using convolutional neural networks. AgriEngineering, 1(1): 119-131. doi:10.3390/agriengineering1010009.

Sokolova, M., Lapalme, G., 2009. A systematic analysis of performance measures for classification tasks. Information Processing \& Management, 45(4): 427-437. doi:10.1016/j.ipm.2009.03.002.

Teja, B. S. M., Anita, C. S., Rajalakshmi, D., Berlin, M. A., 2020. A CNN based facial expression recognizer. Materials Today: Proceedings, 37(2): 2578-2581. doi:10.1016/j.matpr.2020.08.501.

Too, E.C., Yujian, L., Njuki, S., Yingchun, L., 2019. A comparative study of fine-tuning deep learning models for plant disease identification. Computers and Electronics in Agriculture, 161: 272-279. doi:10.1016/j.compag.2018.03.032.

Wang, Z. R., Du, J., 2021. Joint architecture and knowledge distillation in CNN for Chinese text recognition. Pattern Recognition, 111:107722. doi:10.1016/j.patcog.2020.107722.

Waheed, A., Goyal, M., Gupta, D., Khanna, A., Hassanien, A. E., Pandey, H. M., 2020. An optimized dense convolutional neural network model for disease recognition and classification in corn leaf. Computers and Electronics in Agriculture, 175:105456. doi:10.1016/j.compag.2020.105456

Zeng, L., Sun, B., Zhu, D., 2021. Underwater target detection based on Faster R-CNN and adversarial occlusion network. Engineering Applications of Artificial Intelligence, 100:104190. doi:10.1016/j.engappai.2021.104190.

Zhao, D., Ma, H., Yang, Z., Li, J., Tian, W., 2020. Finger vein recognition based on lightweight CNN combining center loss and dynamic regularization. Infrared Physics \& Technology, 105:103221. doi:10.1016/j.infrared.2020.103221.

Zhou, G., Zhang, W., Chen, A., He, M., Ma, X., 2019. Rapid detection of rice disease based on FCM-KM and faster R-CNN fusion. IEEE Access, 7:143190-143206. doi:10.1109/ACCESS.2019.2943454 